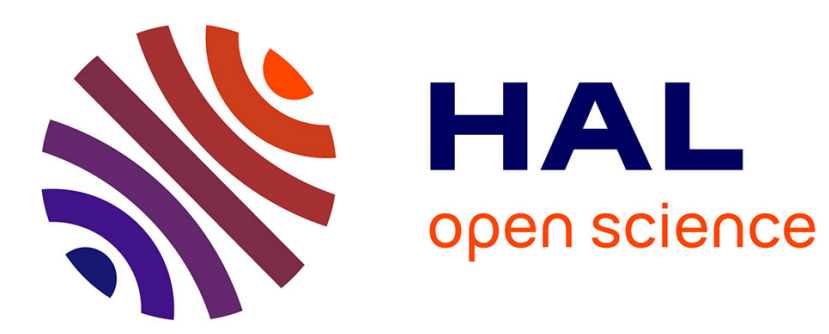

\title{
The Minimal Graph Model of Lambda Calculus
}

\author{
Antonio Bucciarelli, Antonino Salibra
}

\section{To cite this version:}

Antonio Bucciarelli, Antonino Salibra. The Minimal Graph Model of Lambda Calculus. Mathematical Foundations of Computer Science, 2003, Bratislava, Slovakia. pp.300-307. hal-00149557

\section{HAL Id: hal-00149557 https://hal.science/hal-00149557}

Submitted on 26 May 2007

HAL is a multi-disciplinary open access archive for the deposit and dissemination of scientific research documents, whether they are published or not. The documents may come from teaching and research institutions in France or abroad, or from public or private research centers.
L'archive ouverte pluridisciplinaire HAL, est destinée au dépôt et à la diffusion de documents scientifiques de niveau recherche, publiés ou non, émanant des établissements d'enseignement et de recherche français ou étrangers, des laboratoires publics ou privés. 


\title{
The Minimal Graph Model of Lambda Calculus
}

\author{
Antonio Bucciarelli ${ }^{1}$ and Antonino Salibra ${ }^{2}$ \\ 1 Université Paris 7, Equipe PPS, \\ 2 place Jussieu, 72251 Paris Cedex 05, France \\ buccia@pps.jussieu.fr, \\ $2 \star \star \star$ Università Ca'Foscari di Venezia, Dipartimento di Informatica \\ Via Torino 155, 30172 Venezia, Italia \\ salibra@dsi.unive.it
}

\begin{abstract}
A longstanding open problem in lambda-calculus, raised by G.Plotkin, is whether there exists a continuous model of the untyped lambda-calculus whose theory is exactly the beta-theory or the beta-eta-theory. A related question, raised recently by C.Berline, is whether, given a class of lambda-models, there is a minimal theory represented by it.

In this paper, we give a positive answer to this latter question for the class of graph models à la Plotkin-Scott-Engeler. In particular, we build a graph model in which the equations satisfied are exactly those satisfied in any graph model.
\end{abstract}

\section{Introduction}

Lambda theories (i.e., congruence relations on $\lambda$-terms closed under $\alpha$-and $\beta$-conversion) are equational extensions of the untyped lambda calculus that are closed under derivation. Lambda theories arise by syntactical considerations, a lambda theory may correspond to a possible operational (observational) semantics of the lambda calculus, as well as by semantic ones, a lambda theory may be induced by a model of lambda calculus through the kernel congruence relation of the interpretation function (see e.g. [4], [7]). Since the lattice of the lambda theories is a very rich and complex structure, syntactical techniques are usually diffi cult to use in the study of lambda theories. Therefore, semantic methods have been extensively investigated.

Computational motivations and intuitions justify Scott's view of models as partially ordered sets with a least element and of computable functions as monotonic functions over these sets. After Scott, mathematical models of the lambda calculus in various categories of domains were classifi ed into semantics according to the nature of their representable functions (see e.g. [4], [7], [17]). Scott's continuous semantics [19] is given in the category whose objects are complete partial orders (cpo's) and morphisms are Scott continuous functions. The stable semantics introduced by Berry [8] and the strongly stable semantics introduced by Bucciarelli-Ehrhard [9] are strengthenings of the continuous semantics. The stable semantics is given in the category of DI-domains with stable functions as morphisms, while the strongly stable one is given in the category of DI-domains with coherence, and strongly stable functions as morphisms. All these semantics are structurally and equationally rich in the sense that it is possible to build up $2^{\aleph_{0}}$ models in each of them inducing pairwise distinct lambda theories [14] [15].

\footnotetext{
${ }^{\star \star}$ Partially supported by MURST Cofin'01 COMETA Project and by a visiting fellowship granted by the Equipe
} PPS of the University Paris 7-Denis Diderot. 
The following are long standing open problems in lambda calculus (see Berline [7]):

Question 1. Is there a continuous (stable, strongly stable) model whose theory is exactly $\lambda \beta$ or $\lambda \beta \eta$ (where $\lambda \beta$ is the minimal lambda theory and $\lambda \beta \eta$ is the minimal extensional lambda theory)?

Question 1 can be weakened in two ways:

Question 2. Is $\lambda \beta$ the intersection of all theories of continuous semantics? and similarly for $\lambda \beta \eta$ and extensional models; and similarly for other semantics.

A lambda theory $T$ is the minimal theory of a class $\mathcal{C}$ of models if there exists a model in the class which induces $T$, while all the other models in the class induce theories including $T$.

Question 3. Given a class of models in a given semantics, is there a minimal lambda theory represented in it?

Two related question have been answered. Given a semantics, it is natural to ask if all possible $\lambda$-theories are induced by a model in the semantics. Negative answers to this question for the continuous, stable and strongly stable semantics were obtained respectively by HonsellRonchi della Rocca [13], Bastonero-Gouy [6] and Salibra [18]. All the known semantics are thus incomplete for arbitrary lambda theories. On the other hand, Di Gianantonio et al. [12] have shown that $\lambda \beta \eta$ can arise as the theory of a model in the $\omega_{1}$-semantics (thus all questions collapse and have a positive answer in this case). If $\omega_{0}$ and $\omega_{1}$ denote, respectively, the fi rst infi nite ordinal and the fi rst uncountable ordinal, then the models in the co-semantics are the reflexive objects in the category whose objects are $\omega_{0}$ - and $\omega_{1}$-complete partial orders, and whose morphisms preserve limits of $\omega_{1}$-chains (but not necessarily of $\omega_{0}$-chains).

Another result of [12] is that Question 3 admits a positive answer for Scott's continuous semantics, at least if we restrict to extensional models. However, the proofs of [12] use logical relations, and since logical relations do not allow to distinguish terms with the same applicative behavior, the proofs do not carry out to non-extensional models.

Among the set-theoretical models of the untyped lambda calculus that were introduced in the seventies and early eighties, there is a class whose members are particularly easy to describe (see Section 2 below). These models, referred to as graph models, were isolated by Plotkin, Scott and Engeler [4] within the continuous semantics. Graph models have been proved useful for giving proofs of consistency of extensions of $\lambda$-calculus and for studying operational features of $\lambda$-calculus. For example, the simplest of all graph models, namely Engeler-Plotkin's model, has been used to give concise proofs of the head-normalization theorem and of the leftnormalization theorem of $\lambda$-calculus (see [7]), while a semantical proof based on graph models of the "easiness" of $(\lambda x . x x)(\lambda x . x x)$ was obtained by Baeten and Boerboom in [3]

Intersection types were introduced by Dezani and Coppo [10] to overcome the limitations of Curry's type discipline. They provide a very expressive type language which allows to describe and capture various properties of $\lambda$-terms. By duality, type theories give rise to fi lter models of lambda calculus (see [1], [5]). Di Gianantonio and Honsell [11] have shown that graph models are strictly related to fi lter models, since the class of $\lambda$-theories induced by graph models is included in the class of $\lambda$-theories induced by non-extensional fi lter models. Alessi et 
al. [2] have shown that this inclusion is strict, namely there exists an equation between $\lambda$-terms which cannot be proved in any graph model, whilst this is possible with non-extensional fi lter models.

In this paper we show that the graph models admit a minimal lambda theory. This result provides a positive answer to Question 3 for the restricted class of graph models. An interesting question arises: what equations between $\lambda$-terms are equated by this minimal lambda theory?

The answer to this diffi cult question is still unknown; we conjecture that the right answer is the minimal lambda theory $\lambda \beta$. By what we said in the previous paragraph this would solve the same problem for the class of fi lter models. We conclude this introduction by giving a sketch of the technicalities used in the proof of the main theorem. For any equation between $\lambda$-terms which fails in some graph model we fi $x$ a graph model, where the equation fails. Then we use a technique of completion for gluing together all these models in a unique graph model. Finally, we show that the equational theory of this completion is the minimal lambda theory of graph models.

\section{Graph models}

To keep this article self-contained, we summarize some defi nitions and results concerning graph models that we need in the subsequent part of the paper. With regard to the lambda calculus we follow the notation and terminology of [4].

The class of graph models belongs to Scott's continuous semantics. Historically, the fi rst graph model was Plotkin and Scott's $P_{\omega}$, which is also known in the literature as "the graph model". "Graph" referred to the fact that the continuous functions were encoded in the model via (a suffi cient fragment of) their graph.

As a matter of notation, for every set $D, D^{*}$ is the set of all fi nite subsets of $D$, while $\mathcal{P}(D)$ is the powerset of $D$.

Definition 1. A graph model is a pair $(D, p)$, where $D$ is an infinite set and $p: D^{*} \times D \rightarrow D$ is an injective total function.

Let $(D, p)$ be a graph model and $E n v_{D}$ be the set of $D$-environments $\rho$ mapping the set of the variables of $\lambda$-calculus into $\mathcal{P}(D)$. We defi ne the interpretation $M^{p}: E n v_{D} \rightarrow \mathcal{P}(D)$ of a $\lambda$-term $M$ as follows.

$$
\begin{aligned}
& \text { - } x_{\rho}^{p}=\rho(x) \\
& \text { - }(M N)_{\rho}^{p}=\left\{\alpha \in D \mid \exists a \subseteq N_{\rho}^{p} \text { s.t. } p(a, \alpha) \in M_{\rho}^{p}\right\} \\
& \text { - }(\lambda x . M)_{\rho}^{p}=\left\{p(a, \alpha) \mid \alpha \in M_{\rho[x:=a]}^{p}\right\}
\end{aligned}
$$

It is not diffi cult to show that any graph model $(D, p)$ is a model of $\beta$-conversion, i.e., it satisfi es the following condition:

$$
\lambda \beta \vdash M=N \Rightarrow M_{\rho}^{p}=N_{\rho}^{p} \text {, for all } \lambda \text {-terms } M, N \text { and all environments } \rho \text {. }
$$


Then any graph model $(D, p)$ defi nes a model for the untyped lambda calculus through the reflexive cpo $(\mathcal{P}(D), \subseteq)$ determined by the continuous (w.r.t. the Scott topology) mappings

$$
F: \mathcal{P}(D) \rightarrow[\mathcal{P}(D) \rightarrow \mathcal{P}(D)] ; \quad G:[\mathcal{P}(D) \rightarrow \mathcal{P}(D)] \rightarrow \mathcal{P}(D)
$$

defi ned by

$$
F(X)(Y)=\{\alpha \in D:(\exists a \subseteq Y) p(a, \alpha) \in X\} ; \quad G(f)=\left\{p(a, \alpha): \alpha \in f(a), a \in D^{*}\right\},
$$

where $[\mathcal{P}(D) \rightarrow \mathcal{P}(D)]$ denotes the set of continuous selfmaps of $\mathcal{P}(D)$. For more details we refer the reader to Berline [7] and to Chapter 5 of Barendregt's book [4].

Given a graph model $(D, p)$, we say that $M^{p}=N^{p}$ if, and only if, $M_{\rho}^{p}=N_{\rho}^{p}$ for all environments $\rho$. The lambda theory $T h(D, p)$ induced by $(D, p)$ is defi ned as

$$
T h(D, p)=\left\{M=N: M^{p}=N^{p}\right\} .
$$

It is well known that $T h(D, p)$ is never extensional because $(\lambda x . x)^{p} \neq(\lambda x y . x y)^{p}$. Given this huge amount of graph models (one for each total pair $(D, p)$ ), one naturally asks how many different lambda theories are induced by these models. Kerth has shown in [14] that there exist $2^{\aleph_{0}}$ graph models with different lambda theories.

A lambda theory $T$ is the minimal lambda theory of the class of graph models if there exists a graph model $(D, p)$ such that $T=T h(D, p)$ and $T \subseteq T h(E, i)$ for all other graph models $(E, i)$.

The completion method for building graph models from "partial pairs" was initiated by Longo in [16] and recently developed on a wide scale by Kerth in [14] [15]. This method is useful to build models satisfying prescribed constraints, such as domain equations and inequations, and it is particularly convenient for dealing with the equational theories of the graph models.

Definition 2. A partial pair $(D, p)$ is given by an infinite set $D$ and a partial, injective function $p: D^{*} \times D \rightarrow D$.

A partial pair is a graph model if and only if $p$ is total. We always suppose that no element of $D$ is a pair. This is not restrictive because partial pairs can be considered up to isomorphism.

Definition 3. Let $(D, p)$ be a partial pair. The Engeler completion of $(D, p)$ is the graph model $(E, i)$ defined as follows:

- $E=\bigcup_{n \in \omega} E_{n}$, where $E_{0}=D, E_{n+1}=E_{n} \cup\left(\left(E_{n}^{*} \times E_{n}\right)-\operatorname{dom}(p)\right)$.

- Given $a \in E^{*}, \alpha \in E$,

$$
i(a, \alpha)=\left\{\begin{array}{l}
p(a, \alpha) \text { if } a \cup\{\alpha\} \subseteq D, \text { and } p(a, \alpha) \text { is defined } \\
(a, \alpha) \text { otherwise }
\end{array}\right.
$$


It is easy to check that the Engeler completion of a given partial pair $(D, p)$ is actually a graph model. The Engeler completion of a total pair $(D, p)$ is equal to $(D, p)$.

A notion of rank can be naturally defi ned on the Engeler completion $(E, i)$ of a partial pair $(D, p)$. The elements of $D$ are the elements of rank 0 , while an element $\alpha \in E-D$ has rank n if $\alpha \in E_{n}$ and $\alpha \notin E_{n-1}$.

We conclude this preliminary Section by remarking that the classic graph models, such as Plotkin and Scott's $P_{\omega}$ [4] and Engeler-Plotkin's $\mathcal{E}_{A}$ (with $A$ an arbitrary nonempty set of "atoms") [7], can be viewed as the Engeler completions of suitable partial pairs. In fact, $P_{\omega}$ and $\mathcal{E}$ are respectively isomorphic to the Engeler completions of $(\{0\}, p)$ (with $p(\emptyset, 0)=0)$ and $(A, \emptyset)$.

\section{The minimal graph model}

Let $I$ be the set of equations between $\lambda$-terms which fail to hold in some graph model. For every equation $e \in I$, we consider a fi xed graph model $\left(D_{e}, i_{e}\right)$, where the equation $e$ fails to hold. Without loss of generality, we may assume that $D_{e_{1}} \cap D_{e_{2}}=\emptyset$ for all distinct equations $e_{1}, e_{2} \in I$.

We consider the pair $\left(D_{I}, q_{I}\right)$ defi ned by:

$$
D_{I}=\bigcup_{e \in I} D_{e} ; \quad q_{I}=\bigcup_{e \in I} i_{e} .
$$

This pair fails to be a graph model because the map $q_{I}: D_{I}^{*} \times D_{I} \rightarrow D_{I}$ is not total $\left(q_{I}\right.$ is defi ned only on the pairs $(a, x)$ such that $a \cup\{x\} \subseteq D_{e}$ for some $\left.e \in I\right)$.

Finally, let $(E, i)$ be the Engeler completion of $\left(D_{I}, q_{I}\right)$.

We are going to show that the theory of $(E, i)$ is the intersection of all the theories of graph models, i.e. that:

Theorem 1. The class of graph models admits a minimal lambda theory.

From now on, we focus on one of the $\left(D_{e}, i_{e}\right)$, in order to show that all the equations between closed lambda terms true in $(E, i)$ are true in $\left(D_{e}, i_{e}\right)$.

The idea is to prove that, for all closed $\lambda$-terms $M$

$$
M^{i_{e}}=M^{i} \cap D_{e} .
$$

This takes a structural induction on $M$, and hence the analysis of open terms too. Roughly, we are going to show that equation (1) holds for open terms as well, provided that the environments satisfy a suitable closure property introduced below.

Definition 4. Given $e \in I$, we call $e$-flattening the following function $f_{e}: E \rightarrow E$ defined by induction on the rank of elements of $E$ : 


$$
\begin{aligned}
& \text { if } \operatorname{rank}(x)=0 \text { then } f_{e}(x)=x \\
& \text { if } \operatorname{rank}(x)=n+1 \text { and } x=\left(\left\{y_{1}, \ldots, y_{k}\right\}, y\right) \text { then } \\
& \qquad f_{e}(x)= \begin{cases}i_{e}\left(\left\{f_{e}\left(y_{1}\right), \ldots, f_{e}\left(y_{k}\right)\right\} \cap D_{e}, f_{e}(y)\right) & \text { if } f_{e}(y) \in D_{e} \\
x & \text { otherwise }\end{cases}
\end{aligned}
$$

For all $a \subseteq E, f_{e}(a)$ will denote the set $\left\{f_{e}(x): x \in a\right\}$.

The following easy facts will be useful:

Lemma 1. (a) For all $x \in E$, if $f_{e}(x) \notin D_{e}$ then $f_{e}(x)=x$.

(b) If $a \subseteq E$, $z \in E$ and $f_{e}(z) \in D_{e}$, then $f_{e}(i(a, z))=i_{e}\left(f_{e}(a) \cap D_{e}, f_{e}(z)\right) \in D_{e}$.

We notice that Lemma 1(b) holds, a fortiori, if $z \in D_{e}$.

Definition 5. For $a \subseteq E$ let $\hat{a}=a \cup f_{e}(a)$; we say that $a$ is $e$-closed if $\hat{a}=a$.

Lemma 2. For all $a \subseteq E$, $\hat{a} \cap D_{e}=f_{e}(a) \cap D_{e}$.

Proof. By defi nition, $\hat{a}=a \cup f_{e}(a)$, hence

$\hat{a} \cap D_{e}=\left(a \cap D_{e}\right) \cup\left(f_{e}(a) \cap D_{e}\right)$

Since $f_{e}$ restricted to $D_{e}$ is the identity function, we have

$a \cap D_{e} \subseteq f_{e}(a) \cap D_{e}$, and we are done.

Definition 6. Let $\rho: \operatorname{Var} \rightarrow \mathcal{P}(E)$ be an E-environment. We define the e-restriction $\rho_{e}$ of $\rho$ by $\rho_{e}(x)=\rho(x) \cap D_{e}$, while we say that $\rho$ is e-closed if for every variable $x, \rho(x)$ is e-closed.

The following proposition is the key technical lemma of the paper:

Proposition 1. Let $M$ be a $\lambda$-term and $\rho$ be an e-closed E-environment; then

(i) $M_{\rho}^{i}$ is e-closed.

(ii) $M_{\rho}^{i} \cap D_{e} \subseteq M_{\rho_{e}}^{i}$.

Proof. We prove (i) and (ii) simultaneously by induction on the structure of $M$. If $M \equiv x$, both statements are trivially true.

Let $M \equiv \lambda x . N$, and let us start by proving the statement (i): given $y=i(a, z) \in M_{\rho}^{i}$, we have to show that $f_{e}(y) \in M_{\rho}^{i}$. First we remark that, if $\operatorname{rank}(y)=0$ or $f_{e}(z) \notin D_{e}$, then by Lemma 1(a) $f_{e}(y)=y$ and we are done. Hence, let $y=i(a, z)$ and $f_{e}(z) \in D_{e}$; we have 


$$
\begin{aligned}
& y \in M_{\rho}^{i} \\
& \Rightarrow z \in N_{\rho[x:=a]}^{\rho} \quad \text { by defi nition of }(-)^{i} \\
& \Rightarrow z \in N_{\rho[x:=\hat{a}]}^{i} \quad \text { by monotonicity of }()^{i} \text { w.r.t. environments } \\
& \Rightarrow f_{e}(z) \in N_{\rho[x:=\hat{a}]}^{i} \quad \text { by (i), remark that } \rho[x:=\hat{a}] \text { is closed } \\
& \Rightarrow f_{e}(z) \in N_{(\rho[x:=\hat{a}])_{e}}^{i} \quad \text { by (ii), since } f_{e}(z) \in D_{e}
\end{aligned}
$$

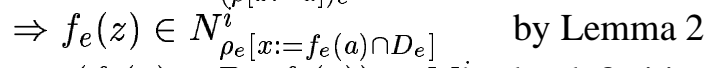

$$
\begin{aligned}
& \Rightarrow i\left(f_{e}(a) \cap D_{e}, f_{e}(z)\right) \in M_{\rho_{e}}^{i} \text { by defi nition of }(-)^{i} \\
& \Rightarrow i_{e}\left(f_{e}(a) \cap D_{e}, f_{e}(z)\right) \in M_{\rho_{e}}^{i} \text { by defi nition of }(E, i) \\
& \Rightarrow f_{e}(y) \in M_{\rho_{e}}^{i} \quad \text { by defi nition of } f_{e} \\
& \Rightarrow f_{e}(y) \in M_{\rho}^{i} \quad \text { by monotonicity of }\left({ }_{-}\right)^{i}
\end{aligned}
$$

Let us prove that $M \equiv \lambda x \cdot N$ satisfi es (ii):

$$
\begin{array}{rlrl} 
& y \in M_{\rho}^{i} \cap D_{e} & \\
\Rightarrow & \left(\exists a \subseteq D_{e}\right)\left(\exists z \in D_{e}\right) y=i_{e}(a, z) \text { and } z \in N_{\rho[x:=a]}^{i} & & \text { by defi nition of }(-)^{i} \text { and by } y \in D_{e} \\
\Rightarrow & z \in N_{(\rho[x:=a])_{e}}^{i} & & \text { by (ii), remark that } \hat{a}=a \\
\Rightarrow & z \in N_{\rho_{e}[x:=a]}^{i} & & \text { since } a \subseteq D_{e} \\
\Rightarrow & y \in M_{\rho_{e}}^{i} & & \text { by defi nition of }(-)^{i}
\end{array}
$$

Let $M \equiv P Q$.

(i) Let $z \in(P Q)_{\rho}^{i}$. If $f_{e}(z)=z$ we are done, otherwise by Lemma 1(a) $f_{e}(z) \in D_{e}$. Moreover, $\exists a \subseteq E$ such that $i(a, z) \in P_{\rho}^{i}$ and $a \subseteq Q_{\rho}^{i}$. Applying (i) and Lemma 1(b) to $P$ we get

$$
f_{e}(i(a, z))=i_{e}\left(f_{e}(a) \cap D_{e}, f_{e}(z)\right)=i\left(f_{e}(a) \cap D_{e}, f_{e}(z)\right) \in P_{\rho}^{i}
$$

Applying (i) to $Q$ we get $f_{e}(a) \subseteq Q_{\rho}^{i}$. Hence $f_{e}(z) \in M_{\rho}^{i}$.

(ii) If $z \in(P Q)_{\rho}^{i} \cap D_{e}$, then $\exists a \subseteq E$ such that $i(a, z) \in P_{\rho}^{i}$ and $a \subseteq Q_{\rho}^{i}$. Since $\rho$ is $e$-closed and $z \in D_{e}$, then by (i) and by Lemma 1(b) we get $f_{e}(i(a, z))=i_{e}\left(f_{e}(a) \cap D_{e}, z\right) \in P_{\rho}^{i}$ and $f_{e}(a) \cap D_{e} \subseteq Q_{\rho}^{i}$. Now, by (ii), we obtain $i_{e}\left(f_{e}(a) \cap D_{e}, z\right) \in P_{\rho_{e}}^{i}$ and $f_{e}(a) \cap D_{e} \subseteq Q_{\rho_{e}}^{i}$, and we conclude $z \in(P Q)_{\rho_{e}}^{i}$.

Proposition 2. Let $M$ be a $\lambda$-term and $\rho: \operatorname{Var} \rightarrow \mathcal{P}\left(D_{e}\right)$ be a $D_{e}$-environment; then we have $M_{\rho}^{i} \cap D_{e}=M_{\rho}^{i_{e}}$.

Proof. We prove by induction on the structure of $M$ that $M_{\rho}^{i} \cap D_{e} \subseteq M_{\rho}^{i_{e}}$. The converse is ensured by $M_{\rho}^{i_{e}} \subseteq M_{\rho}^{i}$ and $M_{\rho}^{i_{e}} \subseteq D_{e}$, both trivially true.

If $M \equiv x$, the statement trivially holds.

Let $M \equiv \lambda x N$; if $y \in M_{\rho}^{i} \cap D_{e}$, then $y=i_{e}(a, z)$ with $a \cup\{z\} \subseteq D_{e}$, and $z \in N_{\rho[x:=a]}^{i}$. By induction hypothesis $z \in N_{\rho[x:=a]}^{i_{e}}$, and hence $i_{e}(a, z)=y \in M_{\rho}^{i_{e}}$. 
Let $M \equiv P Q$; If $z \in(P Q)_{\rho}^{i} \cap D_{e}$, then $\exists a \subseteq E$ such that $i(a, z) \in P_{\rho}^{i}$ and $a \subseteq Q_{\rho}^{i}$. Since $\rho$ is $e$-closed and $z \in D_{e}$, we can use Lemma 1(b) and Proposition 1(i) to obtain

$$
f_{e}(i(a, z))=i_{e}\left(f_{e}(a) \cap D_{e}, z\right) \in P_{\rho}^{i} .
$$

Hence we can use the induction hypothesis to get $i_{e}\left(f_{e}(a) \cap D_{e}, z\right) \in P_{\rho}^{i_{e}}$. Moreover, $f_{e}(a) \cap$ $D_{e} \subseteq Q_{\rho}^{i_{e}}$ by using again Proposition 1(i) and the induction hypothesis on $Q$. Hence $z \in$ $(P Q)_{\rho}^{i_{e}}$.

Proposition 3. $T h(E, i) \subseteq T h\left(D_{e}, i_{e}\right)$.

Proof. Let $M^{i}=N^{i}$. By the previous proposition we have

$$
M^{i_{e}}=M^{i} \cap D_{e}=N^{i} \cap D_{e}=N^{i_{e}} .
$$

Theorem 1 is an immediate corollary of Proposition 3 and of the defi nition of $(E, i)$.

\section{Conclusion}

We have shown that the graph models admit a minimal lambda theory $T h(E, i)$.

Graph models provide a suitable framework for proving the consistency of extensions of $\lambda \beta$. For instance, for every closed $\lambda$-term $M$ there exists a graph model $\left(D_{M}, i_{M}\right)$ such that $(\lambda x . x x)(\lambda x . x x)^{i_{M}}=M^{i_{M}}$ [3]. Symmetrically, one could use them in order to realise inequalities between non $\beta$-equivalent terms: given $M \neq \beta \quad$, this can be achieved by fi nding a graph model $(D, i)$ such that $M^{i} \neq N^{i}$. We are not able to perform this construction in general, yet, but we conjecture that $T h(E, i)=\lambda \beta$.

Another question raised by this work concerns the generality of the notions of $e$-flattening and $e$-closure, introduced to prove the minimality of $(E, i)$. Actually it should be possible to apply our technique for proving that classes of models other than graph models, which, informally, are closed under direct product of "pre-models" and free completion, admit a minimal lambda theory.

\section{References}

1. Abramsky, S.: Domain theory in logical form. Annals of Pure and Applied Logic 51 (1991) 1-77

2. Alessi, F., Dezani, M., Honsell, F.: Filter models and easy terms. in ICTCS'01, LNCS 2202, Springer-Verlag, (2001) 17-37

3. Baeten, J., Boerboom, B.: Omega can be anything it should not be. Indag. Mathematicae 41 (1979) 111-120

4. Barendregt, H.P.: The lambda calculus: Its syntax and semantics. Revised edition, Studies in Logic and the Foundations of Mathematics 103, North-Holland Publishing Co., Amsterdam (1984)

5. Barendregt, H.P., Coppo, M., Dezani, M.: A filter lambda model and the completeness of type assignment. J. Symbolic Logic 48 (1983) 931-940

6. Bastonero, O., Gouy, X.: Strong stability and the incompleteness of stable models of $\lambda$-calculus. Annals of Pure and Applied Logic 100 (1999) 247-277 
7. Berline, C.: From computation to foundations via functions and application: The $\lambda$-calculus and its webbed models. Theoretical Computer Science 249 (2000) 81-161

8. Berry, G.: Stable models of typed lambda-calculi. Proc. 5th Int. Coll. on Automata, Languages and Programming, LNCS vol.62, Springer-Verlag (1978)

9. Bucciarelli, A., Ehrhard, T.: Sequentiality and strong stability. Sixth Annual IEEE Symposium on Logic in Computer Science (1991) 138-145

10. Coppo, M., Dezani, M.: An extension of the basic functionality theory for the $\lambda$-calculus. Notre Dame J. Formal Logic 21 (1980) 685-693

11. Di Gianantonio, P., Honsell, F.: An abstract notion of application. in M. Bezem and J.F. Groote, editors, Typed lambda calculi and applications, LNCS 664, Springer-Verlag, (1993) 124-138

12. Di Gianantonio, P., Honsell, F., Plotkin, G.D.: Uncountable limits and the lambda calculus. Nordic J. Comput. 2 (1995) 126-145

13. Honsell, F., Ronchi della Rocca, S.: An approximation theorem for topological $\lambda$-models and the topological incompleteness of $\lambda$-calculus. Journal Computer and System Science 45 (1992) 49-75

14. Kerth, R.: Isomorphism and equational equivalence of continuous lambda models. Studia Logica 61 (1998) 403-415

15. Kerth, R.: On the construction of stable models of $\lambda$-calculus. Theoretical Computer Science $\mathbf{2 6 9}$ (2001)

16. Longo, G.: Set-theoretical models of $\lambda$-calculus: theories, expansions and isomorphisms. Ann. Pure Applied Logic 24 (1983) 153-188

17. Plotkin, G.D.: Set-theoretical and other elementary models of the $\lambda$-calculus. Theoretical Computer Science 121 (1993) 351-409

18. Salibra, A.: Topological incompleteness and order incompleteness of the lambda calculus. ACM Transactions on Computational Logic (2003)

19. Scott, D.S.: Continuous lattices. In: Toposes, Algebraic geometry and Logic (F.W. Lawvere ed.), LNM 274, Springer-Verlag (1972) 97-136 